Cahiers
de a Recherche
sur les Droits Fondamentaux
Cahiers de la recherche sur les droits fondamentaux

$8 \mid 2010$

La liberté d'expression

\title{
Liberté d'expression et convictions religieuses dans la jurisprudence de la Cour européenne des Droits de l'homme
}

\section{Gérard Gonzalez}

\section{(2) OpenEdition Journals}

Édition électronique

URL : https://journals.openedition.org/crdf/6162

DOI : $10.4000 /$ crdf.6162

ISSN : 2264-1246

Éditeur

Presses universitaires de Caen

\section{Édition imprimée}

Date de publication : 20 décembre 2010

Pagination : 91-102

ISBN : 978-2-84133-367-7

ISSN : $1634-8842$

Référence électronique

Gérard Gonzalez, «Liberté d'expression et convictions religieuses dans la jurisprudence de la Cour européenne des Droits de l'homme ", Cahiers de la recherche sur les droits fondamentaux [En ligne], 8 | 2010, mis en ligne le 08 octobre 2020, consulté le 14 novembre 2022. URL : http:// journals.openedition.org/crdf/6162 ; DOI : https://doi.org/10.4000/crdf.6162 


\title{
Liberté d'expression et convictions religieuses dans la jurisprudence de la Cour européenne des Droits de l'homme
}

\author{
Gérard GONZALEZ \\ Professeur de droit public à I'Université de Montpellier 1 et à I'Université de Galatasaray (Turquie) \\ IDEDH EA 3976
}

I. La liberté de religion comme censeur de la liberté d'expression

A. Spécificité de la garantie des convictions religieuses

1. Les critères de la garantie spécifique des convictions religieuses

2. L'absence d'exclusivité de la garantie élevée des convictions religieuses

$B$. La garantie raisonnable du respect des convictions religieuses

1. Une grande marge d'appréciation

2. Conséquences mitigées pour la liberté d'expression

II. La liberté d'expression comme vecteur des convictions religieuses

A. L'emprise limitée de l'expression de convictions religieuses sur l'outil de communication

B. L'instrumentalisation contenue de la liberté d'expression au profit de convictions ayant un rapport avec la religion

Dans une société réellement démocratique, les libertés
de l'esprit tiennent une place éminente tant les régimes
autocratiques ou dictatoriaux s'emploient à manipuler la
pensée, à la modeler, voire à l'annihiler. Dès lors, rien
d'étonnant à ce que la liberté d'expression et la liberté de
pensée, de conscience et de religion soient valorisées dans
la «société démocratique» que promeut et défend la
Cour européenne des Droits de l'homme par une juris-
prudence aujourd'hui fournie. Dès l'arrêt Handyside, la
Cour européenne faisait de la liberté d'expression «l'un des fondements essentiels de pareille société (démocratique), l'une des conditions primordiales de son progrès et de l'épanouissement de chacun ${ }^{1}$. Dans ce cadre, la presse (audiovisuelle ou écrite) joue le rôle indispensable de "chien de garde» public $^{2}$ et les journalistes ont une «mission de "chiens de garde" de la démocratie» ${ }^{3}$. La liberté de pensée, de conscience et de religion représente, elle, «l'une des assises d'une "société démocratique" » et

1. Cour EDH, 7 décembre 1976, Handyside c. Royaume-Uni, $\$$ 49, F. Sudre, J.-P. Marguénaud, J. Andriantzimbazovina, A. Gouttenoire et M. Levinet, Les grands arrêts de la Cour européenne des Droits de l'homme, $5^{\mathrm{e}}$ édition, Paris, PUF, 2009, $\mathrm{n}^{\circ} 7$ (ci-après GACEDH).

2. Parmi beaucoup, Cour EDH, 23 septembre 1994, Jersild c. Danemark, $\$ 31$; Cour EDH, 8 octobre 2009, Brunet-Lecomte et Tanant c. France, $\$ 62$.

3. Cour EDH, 7 juin 2007, Dupuis et al. c. France, $\$ 46$. 
«figure, dans sa dimension religieuse, parmi les éléments les plus essentiels de l'identité des croyants et de leur conception de la vie » ${ }^{4}$. La liberté d'expression comme la liberté de pensée, de conscience et de religion contribuent au pluralisme, à la tolérance et à l'esprit d'ouverture qui caractérisent toute société démocratique ${ }^{5}$.

Leur complémentarité saute aux yeux. Pourtant, les chemins empruntés diffèrent et la Convention organise, pour chacune de ces libertés, les parcours qu'elles peuvent emprunter. Mais peuvent-elles, sporadiquement, se croiser, se rejoindre voire se confondre? L'ambivalence de la liberté de religion le donne à penser. Celle-ci relève d'abord du for interne de l'individu et, en tant que telle, est absolue. Nul ne peut me forcer à abdiquer ma foi ni mon attachement à tel système de croyance qui habite mon cœur et mon esprit. Mais elle relève aussi du for externe en ce sens que, heureux d'avoir trouvé le chemin de la vérité, le croyant entend partager sa joie avec ses semblables, coreligionnaires ou futurs convertis à l'occasion de manifestations plus ou moins encadrées. Pour le croyant, l'expression de ses convictions passe par des manifestations spécifiquement énumérées par l'article 9 de la Convention qui dessine un cadre à son exacte mesure. Le culte, l'enseignement, les rites et autres pratiques seront ainsi préservés à l'aune de cette disposition spéciale de la Convention européenne. Mais la liberté d'expression permet d'exprimer des idées, des opinions qui peuvent, elles aussi, refléter certaines convictions. Et si le croyant est incité, lorsqu'il manifeste ses convictions, à se placer sous l'aile protectrice de l'article 9 de la Convention $^{6}$, qu'est-ce qui l'empêche, pour propager ou promouvoir ses convictions religieuses, d'utiliser les facilités que lui confèrent les moyens plus modernes de communication comme la presse, la radio ou la télévision, se plaçant ainsi sous la garantie de l'article 10 ? Et s'il le fait, son message perd-il en cela la spécificité qui lui vaut une protection particulière? L'abaissement du discours religieux au niveau de la simple expression d'opinions ou d'idées nuit-il à la protection accordée? Pour le dire autrement, l'article 10 de la Convention fournit-il une garantie «au rabais» au croyant qui délaisse le terrain privilégié, préparé tout spécialement pour lui, de l'article 9 de la Convention?

D’un autre côté, l'indépendance des protections sous l'empire des articles 9 et 10 de la Convention n'empêche pas la collision des deux libertés. Le parallélisme des deux garanties n'est pas parfait, et la confrontation est parfois inévitable lorsqu'il leur arrive de se croiser. Des limitations de la liberté d'expression sont possibles dans une société démocratique pour protéger notamment «la morale» mais aussi «la réputation et les droits d'autrui » (art. 10, $\$ 2$ ). La morale renvoie, dans une certaine mesure, à la religion et, si la réputation concerne surtout la vie privée, les «droits d'autrui » incluent un certain respect des croyances, au moins dans une mesure qui ne dissuade pas celui qui a de telles croyances, de les avoir ou de les afficher. La Cour rappelle ces limites générales de la liberté d'expression qui intéressent tout particulièrement les situations de confrontation avec les croyances; dès l'arrêt Handyside, elle soulignait que si la liberté d'expression "vaut non seulement pour les "informations" ou "idées" accueillies avec faveur ou considérées comme inoffensives ou indifférentes, mais aussi pour celles qui heurtent, choquent ou inquiètent l'État ou une fraction quelconque de la population, c'est sous réserve des dispositions du paragraphe 2 de l'article 10 ", et que d'un autre côté, «quiconque exerce sa liberté d'expression assume des devoirs et des responsabilités dont l'étendue dépend de sa situation et du procédé technique utilisé » $(\$ 49)$.

L'équilibre n'est jamais facile à trouver lorsqu'il s'agit d'arbitrer entre deux libertés aussi éminentes ${ }^{7}$. Dans quelle mesure, selon la jurisprudence de la Cour, la liberté d'expression doit-elle avoir des égards pour les croyances ayant un fondement religieux? La Cour opère-t-elle une hiérarchisation lorsque liberté d'expression et liberté de croyances entrent en conflit? Si oui, au profit de laquelle de ces deux éminentes libertés?

Réceptacle désigné des idées ou opinions, la liberté d'expression se heurte parfois à une forme de censure dictée par la volonté d'imposer un certain respect des convictions religieuses (I). Par ailleurs, pour certaines annonces, idées ou opinions, certains messages qui ne sont pas suffisamment caractérisés par la transcendance inhérente aux modes classiques de manifestation de la foi, la liberté d'expression peut apparaître comme un vecteur utile (II).

\section{La liberté de religion comme censeur de la liberté d'expression}

La Cour de Strasbourg confère par sa jurisprudence un statut très protecteur à la liberté d'expression, particulièrement la liberté de la presse. Confrontée aux droits d'autrui, celle-ci l'emporte très souvent, qu'il s'agisse par exemple du droit à être protégé contre la discrimination raciale (Cour EDH, 23 septembre 1994, Jersild c. Danemark, $\left.G A C E D H, \mathrm{n}^{\circ} 57\right)$ ou de la protection de l'image et du droit à la réputation au titre de la garantie de la vie privée, même si, sur ce dernier point, un rééquilibrage peut être observé (Cour EDH, 14 juin 2007, Hachette Filipacchi associés $c$. France; Cour EDH, 22 octobre 2007, Otchakovsky-Laurens et Julyc. France). Au contraire, la Cour semble plus sensible

4. Cour EDH, 25 mai 1993, Kokkinakis c. Grèce, $\$ 31$, GACEDH, $\mathrm{n}^{\circ} 54$.

5. Cour EDH, Gde ch., 10 novembre 2005, Leyla Sahin c. Turquie, $\$ 108$.

6. Qui malgré son libellé (liberté de pensée et de conscience) protège mal ou peu les convictions areligieuses, ou en tout cas est rarement invoqué avec succès dans ce domaine. Voir G. Gonzalez, La Convention EDH et la Liberté des religions, Paris, Economica, 1997, p. 15-36; G. Gonzalez, «Les convictions pacifistes peu en cour à Strasbourg ", note sous Cour EDH, 18 décembre 1996, Valsamis c. Grèce, Petites Affiches, nº 64, 1997, p. 33-37.

7. Sur cet équilibre, il faut absolument consulter l'excellent article de P. Rolland, «Existe-t-il un droit au respect des convictions religieuses dans les médias? », RFDA, 2004, p. 1001-1008. Aussi F. Rigaux, « La liberté d'expression et ses limites », RTDH, 1995, p. 401-415. 
aux offenses faites aux croyances religieuses. Mais cette impression est-elle fondée? En réalité, les convictions religieuses ne jouissent que d'une protection mesurée (B) qui amène à relativiser fortement les conséquences de leur garantie spécifique (A).

\section{A. Spécificité de la garantie des convictions religieuses}

La spécificité de la garantie des convictions religieuses repose sur des critères originaux mais n'est pas exclusive.

\section{Les critères de la garantie spécifique des convictions religieuses}

L'élément central de cette spécificité repose sur le caractère "intime» des convictions religieuses. Cette expression apparaît pour la première fois dans l'arrêt Wingrove (25 novembre 1996). Selon la Cour, « [...] une plus grande marge d'appréciation est généralement laissée aux États contractants lorsqu'ils réglementent la liberté d'expression sur des questions susceptibles d'offenser des convictions intimes, dans le domaine de la morale et, spécialement, de la religion » $(\$ 58)$.

La Cour se référera à ce même caractère intime dans les autres affaires mettant en cause une sanction infligée pour dénigrement de croyances religieuses (Cour EDH, 13 septembre 2005, I.A.c. Turquie, $\$ 25 ; 2$ mai 2006, Tatlav c. Turquie, $\$ 29 ; 31$ janvier 2006, Giniewskic. France, $\$ 44)$. Ce critère est aussi présent dans la jurisprudence de la Cour relative aux limites de la liberté d'expression imposées par le respect de la vie privée. Par exemple, dans l'arrêt Ici Paris, la Cour note que «les éléments d'information concernant la manière dont l'intéressé gérait et dépensait généreusement son argent, ne relevaient pas du cercle intime de la vie privée protégée par l'article 8 de la Convention $»^{8}$.

Dans le champ des convictions religieuses toutefois, l'intimité dont il est question est d'une tout autre nature. Relative à des convictions spirituelles, elle puise sa spécificité au plus profond de l'âme. Difficile à mesurer, impossible à apprécier, elle mérite d'être préservée dans des proportions qui peuvent passer pour exorbitantes.

L'autre composante de cette spécificité réside dans la difficulté à cerner la notion de religion, comme les moyens spécifiques (l'incrimination de blasphèmes) encore utilisés dans certains États pour la protéger d'attaques offensantes. Selon la Cour, «comme pour la morale, il n'est pas possible de discerner à travers l'Europe une conception uniforme de la signification de la religion dans la société ; semblables conceptions peuvent même varier au sein d'un seul pays. Pour cette raison, il n'est pas possible d'arriver à une définition exhaustive de ce qui constitue une atteinte admissible au droit à la liberté d'expression lorsque celui-ci s'exerce contre les sentiments religieux d'autrui » (Cour EDH, 20 septembre 1994, Otto-Preminger c. Autriche, $\$ 50)$.

La Cour juge compatible avec la Convention l'application d'une législation sur le blasphème à la projection d'un film (Le Concile d'amour) constituant une attaque injurieuse contre des objets de vénération religieuse. Dans l'arrêt Wingrove, la Cour applique le même raisonnement à la sanction spécifique du blasphème: «il n’y a pas encore, dans les ordres juridiques et sociaux des États membres du Conseil de l'Europe, une concordance de vues suffisante pour conclure qu'un système permettant à un État d'imposer des restrictions à la propagation d'articles réputés blasphématoires n'est pas en soi nécessaire dans une société démocratique, et s'avère par conséquent incompatible avec la Convention» (\$ 57$)$.

La législation sur le blasphème peut être appliquée, sans violer la Convention, à une vidéo offensant " gravement» les chrétiens en mettant en scène sexuellement sainte Thérèse d'Ávila. Dans les affaires plus récentes, la Cour répète cette même pétition de principe sur l'absence de consensus européen dès lors qu'il s'agit de protéger les droits d'autrui en cas d'attaques contre des convictions religieuses ${ }^{9}$.

Le cumul de ces deux caractéristiques fonde la spécificité de la protection des croyances religieuses et aboutit à conférer une très large marge d'appréciation aux États qui entendent protéger ces croyances contre les débordements de la liberté d'expression. Cela ne veut bien sûr pas dire que toute critique à l'égard des croyances religieuses est exclue. La Cour contrôle le rapport de proportionnalité entre l'offense incriminée et la sanction adoptée mais, plus que dans d'autres domaines, la Cour considère que compte tenu de ces particularités cumulées, les États sont mieux placés qu'elle pour apprécier les limitations à apporter à la liberté d'expression s'attaquant aux croyances religieuses. Son contrôle est alors un contrôle minimum, un contrôle de l'erreur manifeste d'appréciation des autorités étatiques qui peut parfois aboutir à la sanction d'une ingérence gravement disproportionnée dans l'exercice de la liberté d'expression ${ }^{10}$. Ce contrôle prend en considération un certain nombre de critères.

Le contexte de la confrontation est déterminant. De tous les modes d'expression, la liberté d'expression artistique paraît bien la plus exposée. La Cour semble considérer comme un postulat qu'une large publicité lui est assurée (affaires Müller, Otto-Preminger, Wingrove). Sur la vidéo notamment, la Cour notait en 1996 « qu’il est de la nature des films vidéo qu'une fois mis sur le marché, ils

8. Cour EDH, 23 juillet 2009, Hachette Filipacchi associés (Ici Paris) c. France, $\$ 51$.

9. Cour EDH, 13 septembre 2005 , I.A. c. Turquie: compatibilité avec la Convention de la condamnation du requérant auteur d'un roman contenant des attaques offensantes sur des questions considérées comme sacrées par les musulmans.

10. Cour EDH, 31 janvier 2006, Giniewski c. France, Revue trimestrielle des Droits de l'homme, 2006, p. 839, note de P.-F. Docquir : viole l'article 10 la sanction infligée à l'auteur d'un article ayant évoqué la responsabilité de la doctrine catholique de suprématie de la nouvelle alliance dans l'antisémitisme et, par extension, le génocide juif. Cour EDH, 2 août 2006 , Tatlav $c$. Turquie: viole l'article 10 la sanction infligée à l'auteur d'un ouvrage contenant de vives critiques par rapport à la religion mais nullement insultant. 
peuvent, en pratique, faire l'objet de copie, de prêt, de location, de vente et de projection dans différents foyers, ce qui leur permet d'échapper facilement à toute forme de contrôle par les autorités » et que «dans ces conditions, il n'était pas déraisonnable pour les autorités nationales d'estimer, vu le développement de l'industrie vidéo au Royaume-Uni, que le film pouvait atteindre un public qu'il aurait pu offenser» (Wingrove, \$ 63).

La presse jouit par ricochet d'un haut degré de protection parce qu'elle ne s'adresse pas directement à l'imaginaire fantasmatique mais qu'elle informe, en principe, sur des questions d'intérêt général (Giniewski). Mais une information prodiguée dans un contexte conflictuel au sein d'une Église prend nécessairement une tournure elle-même conflictuelle dont les juridictions nationales doivent tenir compte ${ }^{11}$. L'éthique journalistique interdit cependant une prise de position trop personnelle percluse d'affirmations diffamatoires ${ }^{12}$. Le roman, l'essai, bénéficient aussi d'un préjugé plus favorable. Si les écrits restent, ils marquent moins les esprits que les images (Aydin Tatlav, I.A.). Le degré de publicité attendu ou virtuel surdétermine, dans une certaine mesure, le déroulement du contrôle de la Cour. Dans cette logique, la nature du support de l'expression joue un rôle important. Dans l'affaire Murphy c. Irlande, la Cour soulignait qu'il était « légitime que l'État se méfiât particulièrement des risques d'offense existant dans le contexte de l'audiovisuel, puisque [...] ces médias ont un effet plus immédiat et envahissant et puissant, y compris [...] à l'égard du destinataire passif de l'information ».

Elle se satisfait en l'espèce que le requérant ait été libre de "publier la même annonce dans la presse écrite locale ou nationale, ou à l'occasion de réunions publiques et autres » $(\$ 74)$. Cette affaire était cependant relative à l'interdiction de publicités à caractère religieux, ce qui est un peu différent; mais on peut en conclure que la publicité commerciale directement ou potentiellement offensante peut (et non pas «doit») faire l'objet de mesures restrictives de la part des États ${ }^{13}$.

Le contenu du message délivré tient aussi, bien évidemment, un rôle important. Comme la Cour le répète à l'envi, « ceux qui choisissent d'exercer la liberté de manifester leur religion, qu'ils appartiennent à une majorité ou à une minorité religieuse, ne peuvent raisonnablement s'attendre à le faire à l'abri de toute critique » et « ils doivent tolérer et accepter le rejet par autrui de leurs croyances religieuses et même la propagation par autrui de doctrines hostiles à leur foi» (par exemple Tatlav, $\$ 27$ ). Bien entendu, la critique haineuse d'un groupe religieux ayant un fondement raciste est inadmissible et doit être combattue et lourdement sanctionnée ${ }^{14}$. Mais hors ces situations extrêmes, la tolérance finit aussi lorsque sont formulées des insultes ou des injures à l'égard des croyants ou des symboles sacrés d'une religion (Tatlav, $\$ 28)$ comme « une attaque injurieuse contre la personne du prophète de l'Islam» (I.A., \$29). Les opinions "gratuitement offensantes pour autrui» (Otto-Preminger, $\$ 49$ ) ou profanatrices peuvent être sanctionnées par l'État, mais des «opinions (simplement) offensantes» pour des croyants ne justifient pas forcément une ingérence dans la liberté d'expression (Wingrove, $\$ 60$ ). Une protection spécifique comme l'incrimination de blasphème n'est acceptable que si elle s'applique pour sanctionner un "haut degré de profanation» (Wingrove, $\$ 60$ ). On peut s'inquiéter du caractère hautement subjectif de l'appréciation du caractère injurieux ou du «haut degré» de profanation requis, mais c'est justement le rôle de la Cour de sanctionner les appréciations trop « partisanes » de l'État concerné.

Souvent, c'est la nature de la sanction imposée qui sera déterminante dans le contrôle de proportionnalité de la Cour. S'agissant d'une attaque injurieuse contre le prophète de l'Islam dans un roman, la Cour juge qu'en l'absence de saisie du livre, "la condamnation à une peine d'amende insignifiante» est proportionnée aux buts visés (I.A. c. Turquie, $\$ 32$ ). De façon plus critiquable, l'identification dans le chef de l'expression artistique d'un "haut degré de profanation " propre à une législation sur le blasphème peut justifier des mesures radicales (saisie, confiscation, refus de visa) qui aboutissent à interdire toute diffusion de l'œuvre (affaires Otto-Preminger, Wingrove). Dès lors que le terrain de la pensée religieuse est investi dans le cadre d'un débat d'intérêt général, d'une réflexion historique ou journalistique, toute sanction, aussi minime soit-elle, devient très difficile à justifier au regard du respect dû à la liberté d'expression (affaires Giniewski; Tatlav; aussi Cour EDH, 27 février 2001, Susan Jérusalem; Cour EDH, 22 décembre 2005, Paturel c. France).

Tous ces ingrédients sont mixés par la Cour de façon à faire apparaître si l'ingérence dans la liberté d'expression obéissait bien à "un besoin social impérieux» (Wingrove, $\$ 58 ;$ Murphy, \$67; Tatlav, \$25; Giniewski, $\$ 44)$.

11. Cour EDH, 19 janvier 2006, Albert-Engelmann-Geselschaft MBH c. Autriche: publication par un journal catholique Der 13 de lettres critiques à l'égard d'un dignitaire de l'épiscopat autrichien qualifié de « rebelle», ayant dénigré le pape. Intervenu dans un débat religieux relatif à la nomination de l'archevêque de Salzbourg revêtant " un intérêt considérable " pour la communauté catholique à l'époque, la sanction de ces « jugements de valeur » non dénués de base factuelle viole l'article 10.

12. Cour EDH, déc. 3 avril 2003, Inna Harlanova c. Lettonie: non-violation de l'article 10 du fait de la condamnation pour diffamation d'une journaliste ayant repris à son compte de graves accusations non avérées dans le cadre d'un conflit divisant l'Église orthodoxe du pays.

13. Qui peuvent avoir des difficultés à l'égard de messages diffusés via Internet: Cour EDH, déc. 13 novembre 2007, Muscio c. Italie: on ne peut reprocher à l'État son inaction pour protéger ce catholique convaincu d'e-mails pornographiques dont il est impossible de déterminer l'origine.

14. Cour EDH, 10 juillet 2008, Soulas c. France: la critique virulente de l'immigration et des communautés musulmanes dans un livre encourt la sanction des tribunaux nationaux et de fortes amendes sans violer l'article 10. Même la distribution de tracts injurieux pour une communauté ethnique par un responsable politique encourt pareilles sanctions (Cour EDH, 16 juillet 2009, Féret c. Belgique : tracts virulents s'opposant notamment à l'« islamisation de la Belgique»). Les propos sanctionnés peuvent parfois échapper au contrôle de la Cour s'ils relèvent de l'article 17 de la Convention (par exemple, Cour EDH, déc. 2 février 2007, Pavel Ivanov c. Russie: attaques contre les juifs qualifiés de «groupe ethnique malfaisant»). 


\section{L'absence d'exclusivité de la garantie élevée des convictions religieuses}

Dès ce stade, il est possible de relever que la prétendue spécificité de la protection accordée aux convictions religieuses n'est pas si exclusive qu'il y paraît. Tout d'abord, la protection de la morale en général, non directement reliée à des croyances religieuses, confère à l'État la même large marge d'appréciation compte tenu de l'absence de consensus en ce domaine ${ }^{15}$. Ainsi, même déconnectée des croyances religieuses, à laquelle on peut penser qu' elle est néanmoins implicitement liée, la morale, notion changeante autant dans l'espace que dans le temps, donne à l’État une grande liberté pour museler certaines formes de la liberté d'expression, notamment artistique dans l'affaire Müller ${ }^{16}$. Par ailleurs, la marge d'appréciation reconnue aux États dans le cadre de l'atteinte aux convictions religieuses ne surclasse en rien la large protection accordée aux assertions qui trouvent à s'insérer dans un débat d'idées, d'intérêt général, de dimension historique, sociétale ou religieuse. Dans ce cadre-là, il est possible de considérer que la marge d'appréciation des États s'amenuise considérablement. L'attaque, la provocation, ne peuvent être sanctionnées dès lors qu'elles se situent dans le cadre plus large du débat d'idées, qu'il s'agisse « du point de vue critique d'un non-croyant par rapport à la religion sur le terrain sociopolitique » (Tatlav, \$28), d'une " thèse sur la portée d'un dogme et sur ses liens possibles avec les origines de l'Holocauste [...] réflexion que le requérant a voulu exprimer en tant que journaliste et historien» (Giniewski, \$51), d'un débat dans l'arène politique emportant jugements de valeur (Cour EDH, 27 février 2001, Jérusalem $c$. Autriche: qualification d'une association de «psycho-secte ayant un caractère totalitaire »; Cour EDH, 22 décembre 2005, Paturel c. France: dénonciation par un écrivain des dérives des mouvements anti-sectaires privés financés par les pouvoirs publics). Une confirmation récente peut être trouvée dans l'arrêt du 18 septembre 2008, Choudury c. France selon lequel la condamnation pour diffamation du requérant pour avoir soutenu dans une interview à Lyon Mag que le grand Mufti directeur de la Grande Mosquée de Lyon avait une gestion « pas claire» et que « la religion il s'en fout [...] d'ailleurs il n'y connaît rien » viole l'article 10. Les propos sont choquants mais constituent des «jugements de valeur» portant sur des questions d'intérêt général (la gestion d'un lieu de culte), disposant d'une base factuelle suffisante et auxquels un personnage public s'expose. Il est d'ailleurs significatif que, dès lors que l'on se trouve dans le contexte du débat d'idées, la Cour conclut le plus souvent ${ }^{17}$ à l'unanimité à la violation de l'article 10 (Paturel, Giniewski, Tatlav, Choudury). En dehors de ce cadre, les positions sont plus partagées ( 6 voix contre 3 dans Otto-Preminger, 4 contre 3 dans I.A. c. Turquie par exemple). Il faut donc constater qu'au sein de la liberté d'expression, la participation même vigoureusement critique à un débat d'idées bénéficie, comme la protection du respect des croyances religieuses, d'un statut particulier, d'une garantie spécifique justifiée par sa portée et son contenu. La jurisprudence protectrice de cette liberté d'expression là est claire et sans faille et elle bénéficie aussi, d'ailleurs, à l'expression d'opinions ayant aussi un fondement religieux ${ }^{18}$. Il n'en va pas de même en dehors de ce cadre. Mais, si dès les premiers arrêts ayant semblé sanctuariser les convictions religieuses dans le champ de la liberté d'expression, les critiques furent très vives ${ }^{19}$, il semble qu'elles doivent être relativisées aujourd'hui et que, à quelques réserves près, la jurisprudence de la Cour européenne soit devenue justement mesurée, raisonnable.

\section{B. La garantie raisonnable du respect des convictions religieuses}

La jurisprudence de la Cour prête souvent le flanc à la critique, passant pour une démission au profit de l'État mieux placé qu'elle pour apprécier toutes les implications des agressions contre certains idéaux ou dogmes religieux sur son tissu social. Pourtant, la large marge d'appréciation laissée aux États dans ce domaine (1) n'a pas que des conséquences négatives pour la liberté d'expression (2).

\section{Une grande marge d'appréciation}

La marge d'appréciation est la traduction jurisprudentielle du principe de subsidiarité selon lequel les juridictions nationales sont les juges de droit commun de l'application de la Convention (principe de l'épuisement des voies de recours internes). Pour les affaires dont elle est saisie, la Cour ne se départit jamais de cette réserve à l'égard des autorités nationales «mieux placées » qu'elle pour se prononcer sur les contraintes qui ont conduit à restreindre l'exercice d'une liberté. Cette marge d'appréciation permet aussi de sauvegarder le pluralisme européen dès lors que les États agissent au-dessus du commun dénominateur de garantie des droits imposé par la jurisprudence européenne. Elle ne saurait être d'une « uniformité absolue » (Cour EDH, 26 avril 1979, Sunday Times, $\$ 61)$. Dans le domaine de la liberté d'expression confrontée aux croyances religieuses, la marge d'appréciation est

15. Affaire Handyside, $\$ 48$ (validation par la Cour de la saisie d'un ouvrage destiné aux écoliers jugé subversif sur le plan de l'autorité et de la liberté sexuelle et poursuite de son directeur au pénal); Cour EDH, 24 mai 1988, Müller c. Suisse, $\$ 35, G A C E D H, n^{\circ} 58$ (validation de la saisie de tableaux d'une exposition représentant crûment des relations sexuelles, en particulier entre hommes et animaux).

16. Voir aussi Cour EDH, déc. 22 juin 2006, V.D. \& C.G. c. France: autorisation d'exploitation du film Baise-moi et pour une possible relativisation de cette protection; Cour EDH, 25 janvier 2007, VBK c. Autriche (infra).

17. Il existe des exceptions: Cour EDH, 19 janvier 2006, Albert-Engelmann-Geselschaft MBH c. Autriche: violation à 5 voix contre 2.

18. Voir ci-dessous II. B.

19. Voir, par exemple, F. Rigaux, «La liberté d'expression... »; G. Haarscher, «Le blasphémateur et le raciste », RTDH, 1995, p. 417-422; du même auteur, "Les propos qui heurtent, choquent ou inquiètent : la diffamation collective », Revue de droit, Université libre de Bruxelles, $\mathrm{n}^{\circ} 35,2007$; $\mathrm{P}$. Wachsmann, «La religion contre la liberté d'expression : sur un arrêt regrettable de la Cour EDH », RUDH, 1994, p. 441 sq. 
réputée «grande » pour diverses raisons qui se cumulent : nécessité d'équilibrer différents droits protégés ; caractère également éminent des deux libertés concernées ; absence de consensus sur les manières de protéger la morale ou de définir la religion. La Cour, dans le libellé de ses arrêts, et de façon parfois assez maladroite, renforce l'impression que cette marge d'appréciation «grande » devient béante. Par exemple, dans l'affaire Otto-Preminger, la Cour considère que la projection du film litigieux dans un cinéma avec paiement de droit d'entrée et condition d'âge constitue « une expression "suffisamment" publique pour être offensante» $(\$ 54)$. Elle prend aussi en compte «le fait que la religion catholique romaine est celle de l'immense majorité des Tyroliens » $(\$ 55)$. Les termes employés pour contrôler les motifs de l'ingérence démontrent un contrôle limité à l'erreur manifeste: «le contenu du film ne peut passer pour incapable de fonder les conclusions auxquelles les juridictions autrichiennes ont abouti» $(\$ 55)$. La liberté des États de prévoir des infractions spécifiques semble bien aussi aller dans le sens d'une surprotection des convictions religieuses. Dans une décision d'irrecevabilité, la Commission observait que le fait d'ériger le blasphème « en infraction pénale ne suscite en soi aucun doute quant à sa nécessité : si l'on admet que les sentiments religieux du citoyen méritent protection contre les attaques jugées indécentes sur des questions que l'intéressé estime sacrées, on peut alors également juger nécessaire, dans une société démocratique, de stipuler que ces attaques, lorsqu'elles atteignent une certaine gravité, constituent une infraction pénale dont la personne offensée peut saisir le juge ${ }^{20}$. Dans l'affaire Wingrove, l'argument selon lequel la législation sur le blasphème ne concerne, au Royaume-Uni, que la protection de la foi chrétienne fait long feu, la Cour considère que ce «fait incontesté n'enlève rien à la légitimité du but poursuivi » $(\$ 50)$. Paradoxalement cependant, mutatis mutandis, ce constat peut s'avérer par ailleurs protecteur de la liberté d'expression. Ainsi, dans la décision Choudhury ${ }^{21}$, la Commission déclare irrecevable une requête contestant le refus de l'État d'étendre sa législation sur le blasphème pour interdire Les Versets sataniques.

On pourrait multiplier les exemples qui montrent que la Cour opère un contrôle minimum des motifs de façon à se convaincre du caractère «raisonnable» des réponses apportées à un «besoin social impérieux» (par exemple I.A.c. Turquie, $\$ 30$ ). Au total, il apparaît que la dérision essentiellement sexuelle des objets ou de figures centrales d'une religion est la plus exposée, notamment dans le cadre de la liberté d'expression artistique. Encore faut-il qu'il s'agisse de personnages faisant partie intégrante de la doctrine religieuse en question. Les agressions contre des personnages de chair donnent plus de champ à la liberté d'expression. Par exemple dans l'affaire Klein ${ }^{22}$, la Cour juge que la condamnation d'un journaliste à la suite d'un article virulent contenant des allusions sexuelles et des sous-entendus vulgaires à l'égard d'un archevêque ayant pris parti pour l'interdiction du film Larry Flint viole l'article 10 de la Convention.

\section{Conséquences mitigées pour la liberté d'expression}

L'impression est que la Cour européenne garantit mieux le respect de la liberté religieuse au titre des droits d'autrui que la liberté d'expression, notamment artistique, lorsque celle-ci se pique de les attaquer. Mais la Cour peut-elle, en ce domaine, imposer sa propre appréciation à des États au sein desquels les croyances religieuses occupent des places si différentes? En dehors des affaires où elle impose sans faille l'impunité des critiques émises dans un "débat d'idées", l'attitude de la Cour ressemble fort à une démission. La difficulté vient de ce que son raisonnement finaliste sur le fondement des critères déjà présentés apparaît parfois abrupt, trop dépendant des conclusions auxquelles l'État lui-même est parvenu. Il faut donc restituer les conséquences de la large marge d'appréciation laissée aux États dans ce domaine, qui ne sont pas toutes négatives pour la liberté d'expression.

Ainsi, à notre connaissance, la Cour n'a jamais consacré une obligation positive pour les États de sanctionner la liberté d'expression confrontée aux croyances religieuses. Elle n'a été saisie que d'affaires d'ingérences dans la liberté d'expression, mais il n'existe pas d'affaire significative initiée par un requérant invoquant une violation de la Convention au titre de la passivité de l'État à intervenir pour museler la liberté d'expression irrespectueuse à l'égard de ses convictions religieuses ${ }^{23}$. Il faut évoquer toutefois l'affaire $V B K^{24}$. S'agissant d'une peinture osée mettant en scène dans une sorte de frénésie érotique des membres de l'extrême droite autrichienne, un cardinal et mère Teresa, la Cour a rejeté la plainte du requérant, membre de l'extrême droite représenté sur le tableau, en des termes sévères très critiqués par la minorité des juges (4 voix contre 3 ). Doit-on voir dans cette affaire un revirement de jurisprudence de la Cour par rapport aux affaires Müller, Otto-Preminger ou Wingrove? La Cour a, en effet, relevé que les personnages représentés étaient «des caricatures, et la peinture se voulait satirique ». Mais il reste que le requérant n'invoquait pas l'offense à ses croyances religieuses mais la protection de son image, de sa dignité. La solution aurait-elle été identique si des croyants avaient introduit la requête du fait de l'offense à l'Église, en la personne du cardinal ${ }^{25}$, ou à 
mère Teresa? Indirectement, dans l'arrêt Leela Förderkreis ${ }^{26}$, la Cour, statuant sur le fondement de l'article 9 de la Convention, a mis à la charge des États une obligation positive d'informer sur les sectes. Mais il s'agit là d'une action d'information prise directement en charge par l'État et enfermée dans des conditions assez strictes. Quoi qu'il en soit, le résultat global est que l'État est finalement libre d'adopter une politique jurisprudentielle très respectueuse de la liberté d'expression, même lorsqu'elle heurte violemment des croyances religieuses. Les États peuvent sous certaines conditions censurer des expressions injurieuses pour les croyances religieuses, ils ne le doivent pas.

Cette dichotomie fait que la jurisprudence de la Cour semble manquer de lisibilité et désorienter les juridictions nationales; mais, au fond, c'est sans grandes conséquences.

Par exemple, dans une affaire d'interdiction d'une affiche publicitaire représentant la cène, la Cour de cassation $^{27}$, s'appuyant sur le raisonnement de la Cour européenne dans l'affaire Giniewski qui lui avait valu d'être désavouée par la Cour de Strasbourg, a jugé que cette affiche n'était pas constitutive d'une insulte pour les catholiques. Ce faisant, elle ne prend aucun risque puisqu'il n'existe pas d'obligations positives de sanctionner en ce cas la liberté d'expression. Mais elle aurait pu aussi juger le contraire sans doute avec l'assentiment de la Cour européenne dont la jurisprudence est assez sévère à l'égard des démarches commerciales publicitaires mettant en jeu les libertés d'expression et de religion (Cour EDH, 10 juillet 2003, Murphy c. Irlande).

Qu'en est-il des caricatures ou satires? La Cour s'est montrée par le passé sévère (Müller, qui ne concernait pas directement les croyances religieuses; Otto-Preminger). Elle a fait preuve récemment de plus de mansuétude dans l'affaire VBK. Mais, comme cela a été souligné, l'offensé ayant relevé le gant devant les tribunaux était une personnalité politique, les «figures» religieuses brocardées n'ayant intenté aucune action contentieuse. D'ailleurs, quelques semaines auparavant, la Cour s'appuyait sur sa jurisprudence Müller pour déclarer irrecevable la requête contre la «censure» du film Baise-moi ${ }^{28}$. Bien que ne concernant pas directement les croyances religieuses mais la morale au sens large, il faut souligner que le requérant devant la juridiction nationale était une «association pour la promotion des valeurs judéo-chrétiennes ». Que penser de l'affaire des caricatures de Mahomet? Une plainte déposée contre le Danemark a été déclarée irrecevable par la Cour, les requérants ne relevant pas de la «juridiction» de l'État concerné ${ }^{29}$. La Cour a déjà admis une sanction (minime) pour "attaque injurieuse contre la personne du prophète de l'Islam» (I.A. c. Turquie). Elle pourrait donc peut-être avaliser une sanction pour injure si les juridictions nationales, mieux placées qu'elle pour apprécier le contexte de cette affaire, en décidaient ainsi. Mais elle se satisferait sans doute, si elle était saisie, et pour les mêmes raisons, d'une absence de sanction au nom de la liberté d'expression «satirique » en reprenant, par exemple, le raisonnement du TGI de Paris dans l'affaire Charlie Hebdo ${ }^{30}$. La récente affaire Leroy (Cour EDH, 22 octobre 2008), compte tenu de son contexte particulier, n'apporte pas d'éclairage nouveau de ce point de vue. La Cour valide la sanction infligée à l'auteur d'une caricature publiée le 13 septembre 2001 et relative aux attentats du 11 septembre 2001 dans l'hebdomadaire basque Ekaitza avec pour légende: « Nous en avions tous rêvé... le Hamas l'a fait. » La Cour tient compte du court délai depuis l'événement et de la région basque sensible à ce type d'information. La Cour approuve les juridictions internes qui ont vu dans le dessin publié un message apologétique du terrorisme. Il s'agissait ici de magnifier un acte de violence, ce que la Cour condamne régulièrement. Il y a ainsi des constantes dans la jurisprudence de la Cour qui permettent de sérier les questions en fonction de leur contexte et de comprendre les différences d'appréciation qui peuvent être portées. Il est impossible d'appréhender la jurisprudence de la Cour par rapport aux caricatures en général en la déconnectant du contexte particulier dans lequel la provocation s'installe ${ }^{31}$.

S'agissant de la confrontation entre deux libertés aussi éminentes, la jurisprudence de la Cour européenne apparaît finalement assez hésitante. Hors le débat d'idées dont elle a une conception assez large et souple et qui favorise la liberté d'expression, la Cour ne paraît prête qu'à sanctionner les restrictions manifestement abusives de la liberté d'expression, notamment lorsqu'elle prend une connotation sexuelle agressive, ce qui, là encore, favorise la liberté d'expression. Au total, hormis les quelques affaires valorisant en apparence la garantie des croyances religieuses (car encore une fois, il n'existe pas d'obligation positive des États dans ce domaine), c'est la liberté d'expression, et non celle de religion, qui l'emporte. Pourquoi alors ne servirait-elle pas, elle-même, de vecteur de convictions religieuses?

26. Cour EDH, 6 novembre 2008, Leela Förderkreis E.V.c. Allemagne, RTDH, $\mathrm{n}^{\circ}$ 78, 2009, p. 553-568, note G. Gonzalez.

27. Cass. ( $1^{\text {re }}$ civ.), 14 novembre 2006, Gaz. Pal., $\mathrm{n}^{\circ}$ 344-346, p. 9-12, note G. Gonzalez; sur la même affaire en première instance (référé) et en appel, voir note P. Rolland, Recueil Dalloz, ${ }^{\circ}$ 20, 2005, p. 1326-1331.

28. Cour EDH, déc. 22 juin 2006, V.D. \& C.G. c. France.

29. Cour EDH, déc. 11 décembre 2006, Ben El Mahli et al. c. Danemark.

30. TGI Paris, $17^{\mathrm{e}} \mathrm{ch} ., 22$ mars 2007, Soc. Des Habous et des lieux saints de l'Islam et al. c. Philippe Val et soc. Éditions Rotatives, Recueil Dalloz, Actualité jurisprudentielle, 2007, p. 1023; RSC 2007, p. 564, obs. J. Francillon; Dr. pén., 2007, comm. nº6, obs. A. Lepage (confirmé par CA Paris, $11^{\mathrm{e}}$ ch., Section A, 12 mars 2008, nº $07 / 02873$ ).

31. Pour une illustration récente dans le domaine de la «lutte» syndicale: Cour EDH, 8 décembre 2009, Aguilera Jiénez et al. c. Espagne: non-violation de l'article $10 \mathrm{du}$ fait du licenciement de syndicalistes ayant fait paraître dans un bulletin mensuel des caricatures grossièrement offensantes du personnel de direction. 


\section{La liberté d'expression comme vecteur des convictions religieuses}

L'expression de certaines idées ou opinions ayant un fondement religieux peut sortir du cadre délimité par l'article 9 de la Convention pour les manifestations de la liberté de religion. Délaissant le culte, l'enseignement, les rites, les pratiques, le croyant souhaite user de tous les atouts de la modernité et même, parfois, de moyens peu " orthodoxes " pour faire connaître son message, son institution. En Europe, sous le contrôle de la Cour européenne, les États sont plutôt réticents à faciliter l'emprise de l'expression de certaines convictions religieuses sur l'outil de communication, refusant ainsi la contagion du mouvement télévangéliste apparu aux États-Unis dans les années 1980 (A). Par ailleurs, la liberté d'expression doit accueillir l'expression de certaines idées ou opinions qui sont alimentées par des convictions religieuses. Néanmoins, la Cour veille à ce que la liberté d'expression ne soit pas instrumentalisée au profit de convictions ayant un rapport avec des convictions religieuses présentant un danger réel pour la société démocratique qu'elle défend (B).

\section{A. L'emprise limitée de l'expression de convictions religieuses sur l'outil de communication}

L'alinéa premier de l'article 10 "n'empêche pas les États de soumettre les entreprises de radiodiffusion, de cinéma ou de télévision à un régime d'autorisation ». Cela permet d'attribuer les licences de diffusion en fonction de critères techniques, puisque dans ce domaine, les ressources sont limitées, mais aussi en fonction d'autres critères devant être appréciés objectivement. Ainsi, peuvent être prises en compte des « considérations comme celles qui concernent la nature et les objectifs d'une future station, ses possibilités d'insertion au niveau national, régional ou local, les droits et besoins d'un public donné, ainsi que les obligations issues d'instruments juridiques internationaux », autant de verrous qui doivent s'apprécier «à la lumière des exigences du paragraphe 2 " de l'article 10 (Cour EDH, déc. 7 novembre 2000, United Christian Broadcasters Ltd c. Royaume-Uni; Cour EDH, 11 octobre 2007, Glas Nadejda EOOD et Elenkov c. Bulgarie, $\$ 44$ ). La société Glas Nadejda EOOD qui avait sollicité une licence pour diffuser principalement des émissions chrétiennes était en droit d'obtenir de l'autorité de régulation bulgare compétente une certaine transparence. Au lieu de quoi, la décision de refus fut adoptée de façon totalement discrétionnaire sans que le demandeur soit en mesure de connaître la motivation de ce refus même durant l'instance judiciaire relative à la contestation de cette décision. Un tel pouvoir discrétionnaire viole l'article 10 de la Convention. Ainsi, la création de stations ou de chaînes de télévision essentiellement religieuses ne saurait être, en soi, contraire à la Convention mais, dans la marge d'appréciation qui leur est reconnue, les États sont libres de soumettre de telles initiatives à des restrictions, voire à des interdictions, surtout dans un contexte national ou local spécifique. Dans un souci d'égalité, d'équité et de paix sociale, il leur est, par exemple, loisible de refuser toute délivrance de licence de diffusion nationale à des organisations politiques ou religieuses renvoyées à une diffusion seulement locale ${ }^{32}$.

Les mouvements religieux, ou se revendiquant tels, sont aussi souvent éditeurs de revues, brochures destinées à diffuser leur doctrine auprès de leurs adeptes et du grand public. Dans son observation générale $\mathrm{n}^{\circ} 22$ sur l'article 13 PIDCP (Pacte international relatif aux droits civils et politiques) garantissant le droit à la liberté de pensée, de conscience et de religion, le Comité des Droits de l'homme soulignait que «la pratique et l'enseignement de la religion ou de la conviction comprennent les actes indispensables aux groupes religieux pour mener leurs activités essentielles, tels que la liberté de choisir leurs responsables religieux, leurs prêtres et leurs enseignants, celle de fonder des séminaires ou des écoles religieuses, et celle de préparer et de distribuer des textes ou des publications de caractère religieux $"$.

Ainsi, par exemple, le groupement des témoins de Jéhovah est connu pour la distribution des périodiques La Tour de Garde et Réveillez-vous. En règle générale, ces écrits abordent des points de doctrine, de vie pratique et fournissent matière à réflexion aux adeptes et autres lecteurs. Mais l'Église de scientologie a expérimenté un autre usage de cette liberté reconnue aux groupements de diffuser leur littérature. Dans l'un de ses périodiques, elle avait inséré une annonce proposant à la vente l'électromètre Hubbard (E-mètre) dont l'utilisation permettrait à ses acquéreurs, fidèles de l'Église, de mesurer leur foi. Le tribunal du marché suédois avait interdit une partie de la publicité vantant les avantages de cet appareil et insérée dans une revue diffusée parmi les membres du groupe. La censure ne visait que certains passages descriptifs de l'E-mètre, le présentant notamment comme « un moyen précieux de mesurer l'état de l'âme humaine et ses variations ». Saisie de cette censure, la Commission de Strasbourg a constaté que «le tribunal du marché n'a pas empêché l'Église de vendre d'E-mètre, ni même de faire réclame en vue de sa vente ». Mais surtout, elle rappelle que l'article 9 «ne protège pas des professions de prétendue foi religieuse qui apparaissent comme des arguments de vente dans des annonces à caractère purement commercial faites par un groupe religieux ». Selon elle, ces «déclarations à teneur religieuse expriment davantage un désir de commercialiser des marchandises à des fins lucratives qu'une conviction par les pratiques au sens propre du terme» et elles ne sauraient donc entrer dans le cadre de la protection de l'article 9 de la Convention. La vente de certains objets à un prix d'ailleurs exorbitant, alors même qu'il pourrait s'agir «d'objets religieux essentiels au regard d'un besoin particulier», 
révèle l'intention de faire un profit substantiel qui fait sortir le groupement concerné de son rôle au regard de l'article 9 de la Convention. Ayant revêtu l'habit de marchand, il doit être traité comme tel au regard des lois protectrices des consommateurs, fussent-ils ses adeptes. Une activité lucrative d'un organisme religieux ne peut bénéficier de la garantie spécifique de l'article 9 de la Convention tout comme ne peut l'invoquer une société à but lucratif ${ }^{33}$. Le terrain protecteur de l'article 9 étant inapproprié, celui de l'article 10 lui est substitué. La Commission relève d'ailleurs que «les restrictions qui ont été imposées aux annonces des requérants doivent plutôt être examinées sous l'angle de l'article $10{ }^{34}$. Selon elle, l'ingérence tient en ce que les requérants ont "communiqué des idées sur cette opinion (sur le caractère religieux de l'E-mètre) et le tribunal du marché leur a interdit de continuer à employer certains termes ». Cette censure mesurée des «idées» commerciales peut passer pour «nécessaire dans une société démocratique» aux yeux de la Commission. En se dévoyant, le discours religieux perd en protection ce qu'il peut gagner en audience.

Dès lors que le discours de nature religieuse emprunte, pour sa propagation, un support médiatique, la Cour européenne lui accorde la protection garantie par l'article 10 de la Convention plutôt que celle de l'article 9. Le support de propagation du message choisi (un média) déplace ainsi la garantie conventionnelle accordée au discours religieux du terrain de l'article 9 vers celui de l'article 10 de la Convention. Pour déterminer la nature du message, la Cour européenne s'attache plus au fond qu'à la méthode de diffusion. Dans l'affaire Murphy c. Irlande, le Centre irlandais de la foi acheta du temps d'antenne sur une radio locale privée afin d'annoncer la projection d'une vidéo sur le Christ et les preuves de sa résurrection. Selon la Cour, « la nature et le but du message contenu dans l'annonce [...] permettent de le qualifier de religieux, par opposition à commercial, même si le requérant a acheté le temps de diffusion nécessaire ${ }^{35}$. La diffusion fut finalement interdite en vertu d'une disposition législative selon laquelle «aucune annonce à caractère religieux ou politique ou qui se rapporte d'une façon quelconque à un conflit social ne peut être diffusée ». Cette interdiction est jugée proportionnée par la Cour surtout en raison du contexte religieux conflictuel en Irlande. Mais l'arrêt énonce certains principes généraux intéressants quant à la diffusion de messages religieux, et non commerciaux, au regard de la garantie de l'article 10 de la Convention. Tout d'abord, sa réserve, à l'image de la Commission, à l'égard de messages publicitaires, c'est-àdire diffusés lors d'un temps d'antenne acheté et même si leur contenu n'est pas commercial. Partiale par nature, la publicité reposant sur un temps de diffusion acheté « favoriserait une plus grande utilisation de cette prestation par les groupes religieux dotés de ressources plus importantes » et «l'État peut raisonnablement considérer qu'une liberté, même restreinte, de diffuser des annonces favoriserait probablement une religion dominante au détriment des religions qui rassemblent nettement moins d'adeptes et de ressources» $(\text { Murphyc. Irlande, } \$ 74 \text { et } 78)^{36}$.

En revanche, «la présentation de sujets religieux dans les programmes (documentaires, débats, films, discussions et retransmissions en direct d'événements et de manifestations religieux) » est toujours possible en respectant les obligations objectives d'impartialité, de neutralité et d'équilibre. Selon la Cour, «le requérant conservait le même droit que tous les autres citoyens de participer à des programmes portant sur des sujets religieux et de demander que les services célébrés dans son Église fussent diffusés à la radio ou à la télévision » $(\$ 74)$.

D'ailleurs, la Cour a été saisie de requêtes mettant en cause le traitement réservé à ceux qui, porteurs de messages religieux, participent à de tels programmes. Elle veille à éviter une instrumentalisation de la liberté d'expression au profit de convictions ayant un rapport plus ou moins étroit avec la religion.

\section{B. L'instrumentalisation contenue de la liberté d'expression au profit de convictions ayant un rapport avec la religion}

L'expression de convictions se réclamant d'idéaux soi-disant religieux peut être sanctionnée par l'État si elle dépasse certaines limites ou si elle fait la promotion d'un extrémisme incompatible avec une société démocratique. Selon la Cour, « on ne saurait exclure qu'une association, en invoquant les droits consacrés par l'article 11 de la Convention ainsi que par les articles 9 et 10, essaie d'en tirer le droit de se livrer effectivement à des activités

33. Déc. Com. EDH, 27 février 1979, X c. Suisse, DR 16, p. 85-87.

34. Déc. Com. EDH, 5 mai 1979, X et Église de scientologie c. Suède, DR 16, p. 68-81.

35. Cour EDH, 10 juillet 2003, Murphy c. Irlande.

36. La Cour établit une différence dans la portée du message avec l'affaire VGT c. Suisse concernant l'interdiction d'un spot relatif à la protection des animaux et aux méfaits induits par l'expérimentation animale et l'élevage en batterie (arrêt du 28 juin 2001 ; voir aussi l'arrêt de grande chambre du 30 juin 2009 relatif à l'inexécution de ce premier arrêt, \$ 92) trouvé en violation de l'article 10 de la Convention. Selon la Cour, cette affaire portait sur l'interdiction d'une publicité participant à un « débat d'intérêt général », cadre dans lequel la marge d'appréciation des États est réduite. On peut se demander si la nuance est pertinente : le rôle du Christ et la question de sa résurrection sont aussi, d'une certaine manière, des questions relevant d'un débat d'intérêt général dont les participants ne se confrontent pas souvent plus violemment que les défenseurs des animaux avec leurs pourfendeurs (éleveurs, scientifiques ou chasseurs). Faut-il conclure qu'aucun message religieux soulevant un point de doctrine ne peut revendiquer participer à un débat d'intérêt général? Sur le terrain de l'article 10, le message "publicitaire » religieux serait donc, de ce point de vue, nettement moins bien protégé que d'autres opinions ou idées. La justification de la volonté de préserver une forme d'égalité que pourraient corrompre les messages financés par les Églises les plus riches apparaît néanmoins convainquant de ce point de vue mais, là aussi, spécifique aux messages religieux. La Cour cherche en quelque sorte à favoriser une libre concurrence, non faussée, entre les diverses Églises. Voir G. Gonzalez, « Religions et marchands, marchands de religions : arbitrages à la lumière de la Convention européenne des Droits de l'homme », Mélanges en l'honneur d'Yves Serra, Paris, Dalloz, 2006, p. 217-229. 
visant la destruction des droits ou libertés reconnus dans la Convention et ainsi, la fin de la démocratie ${ }^{37}$.

Dans des cas extrêmes, l'article 17 de la Convention peut être un rempart efficace contre l'expression d'idées si abjectes qu'elles ne peuvent en aucun cas s'appuyer sur la garantie offerte par l'article 10 de la Convention ${ }^{38}$. Mais cette disposition couperet ne peut être exagérément utilisée. S'agissant d'un dessin représentant quatre immeubles qui s'effondrent après avoir été percutés par deux avions et accompagné de la légende «Nous en avions tous rêvé... le Hamas l'a fait», la Cour juge que «l'expression litigieuse ne rentre pas dans le champ d'application des publications qui se verraient soustraites par l'article $17 \mathrm{de}$ la Convention à la protection de l'article 10 " comme l'apologie du racisme, de l'antisémitisme ${ }^{39}$ ou de l'islamophobie ${ }^{40}$. Selon elle, "publié sous la forme humoristique certes controversée d'une caricature, le message de fond visé par le requérant - la destruction de l'impérialisme américain - ne vise pas la négation de droits fondamentaux et n'a pas d'égal avec des propos dirigés contre les valeurs qui sous-tendent la Convention [... et] ne constituent pas une justification à ce point non équivoque de l'acte terroriste qui les ferait échapper à la protection garantie par l'article 10 de la liberté de la presse ${ }^{41}$.

Dans ce cas, il reste à la Cour à apprécier «si un juste équilibre a été respecté entre le droit fondamental d'un individu à la liberté d'expression et le droit légitime d'une société démocratique de se protéger contre les agissements d'organisations terroristes $»{ }^{42}$.

En l'espèce, la sanction pécuniaire infligée était bien nécessaire dans une société démocratique. Au contraire, le requérant sanctionné pour avoir apposé la même image à sa fenêtre sous forme de poster appelant ouvertement à chasser tous les musulmans du Royaume-Uni ne peut contester cette ingérence devant la Cour européenne car son action relève bien de la clause de l'article 17 de la Convention ${ }^{43}$.

Dans certains arrêts, la Cour semble accorder à l'expression d'opinions ayant un fondement religieux sur le terrain de l'article 10 une protection spécifique calquée sur celle qui les protège elles-mêmes de toute expression offensante. Ainsi, dans l'affaire Gündüz c. Turquie, la Cour souligne-t-elle au titre des principes généraux applicables: "Quiconque exerce les droits et libertés consacrés au premier paragraphe de cet article assume "des devoirs et des responsabilités". Parmi eux - dans le contexte des opinions et croyances religieuses peut légitimement être comprise une obligation d'éviter autant que faire se peut des expressions qui sont gratuitement offensantes pour autrui et constituent donc une atteinte à ses droits et qui, dès lors, ne contribuent à aucune forme de débat public capable de favoriser le progrès dans les affaires du genre humain (voir, mutatis mutandis, les arrêts Otto-Preminger-Institut c. Autriche, 20 septembre 1994, série $A n^{\circ} 295-A, p .18-19, \$ 49$, et Wingrove c. Royaume-Uni, 25 novembre 1996, \$52). En outre, une certaine marge d'appréciation est généralement laissée aux États contractants lorsqu’ils réglementent la liberté d'expression sur des questions susceptibles d'offenser des convictions intimes, dans le domaine de la morale et, spécialement, de la religion (voir, mutatis mutandis, les arrêts Müller et autres c. Suisse, 24 mai 1988, série $\mathrm{A} \mathrm{n}^{\circ} 133$, p. 22, $\$ 35$, et, en dernier lieu, Murphy $c$. Irlande, $\$ 65-69)$. » ${ }^{44}$

Cette jurisprudence donne à penser que toute expression d'opinions ayant un fondement religieux aboutit à une confrontation avec d'autres opinions relevant forcément de la morale ou de la religion, ce qui revient à dire qu'une opinion ayant un fondement religieux ne pourrait pas être offensante en soi mais uniquement confrontée à une autre opinion de même nature. Il est plus pertinent que la protection accordée aux opinions reposant sur un fondement religieux et émises dans le cadre de l'article 10 de la Convention n'apparaisse pas renforcée par rapport à la protection accordée à d'autres idées. Hors le cadre des manifestations classiques de la liberté religieuse protégées par l'article 9 de la Convention, l'expression d'opinions religieuses se fait le plus souvent par médias interposés, dans le cadre de débats, d'émissions de radio ou de télévision. Dès lors, par ricochet, il semble logique que la protection accordée épouse les contours de la très large liberté reconnue généralement aux organes de presse ou aux discours politiques. Tel est bien d'ailleurs le résultat auquel parvient, parfois confusément, la Cour européenne, notamment quand la dimension religieuse s'insère dans une forme de débat politique. Dans l'affaire Kutlular, le requérant avait été condamné à une peine de prison pour

37. Cour EDH, déc. 11 décembre 2006, Kalifatstaat c. Allemagne. Les implications des articles 9, 10 et 11 de la Convention sont souvent considérées globalement par la Cour qui les combine en privilégiant, selon le contexte, l'un de ces articles à titre principal. Pour les groupements religieux ou politiques, c'est fréquemment l'article 11 qui est choisi à titre principal (voir Cour EDH, Gde ch., 13 février 2003, Refah partisi c. Turquie, GACEDH, ${ }^{\circ} 55$ ) tout comme sont en cause l'organisation de réunions ou celle de manifestations publiques (Cour EDH, 29 juin 2006, Öllinger c. Autriche; 17 juillet 2007, Bukta et al. c. Hongrie; 26 juillet 2007, Barankévitch c. Russie).

38. Cour EDH, déc. 24 juin 2003, Garaudy c. France; Cour EDH, déc. 2 février 2007, Pavel Ivanov c. Russie.

39. Garaudy et Pavel Ivanov.

40. Cour EDH, déc. 16 novembre 2004, Norwood c. R-U; Cour EDH, 10 juillet 2008, Soulas c. France; Cour EDH, 16 juillet 2009 , Féret c. Belgique.

41. Cour EDH, 2 octobre 2008, Leroy c. France, $\$ 27$.

42. Ibid., $\$ 37$.

43. Cour EDH, déc. 16 novembre 2004, Norwood c. R-U. On pourrait voir là une illustration de la protection spécifique des convictions religieuses par rapport à l'affaire Leroy. Dans un cas, l'apologie caricaturale de l'attaque terroriste comme symbole du rejet du modèle américain ne tombe pas sous l'emprise de l'article 17. Dans l'autre, l'utilisation de la même action terroriste pour attaquer un groupe religieux tout entier (les musulmans) tombe sous le coup de l'article 17. Toutefois, il faut noter qu'une attaque tout aussi virulente dans le cadre du débat politique ne relève pas, selon la Cour, de la clause couperet de la Convention (Cour EDH, 16 juillet 2009, Ferets $c$. Belgique, $\$ 82$ ).

44. Cour EDH, 4 décembre 2003, $\$ 37$; Cour EDH, 6 juillet 2006, Erbakan c. Turquie, $\$ 55$; Cour EDH, 29 avril 2008, Kutlular c. Turquie, $\$ 45$. 
avoir, dans le cadre d'une cérémonie religieuse, en réponse à des questions posées par des journalistes, fait le lien entre le séisme de 1999 en Turquie et le manque de foi (non-respect de la prescription du hijab) dans la région la plus touchée par cette catastrophe. La Cour souligne qu' « en conférant une signification religieuse à une catastrophe naturelle et surtout en évoquant un lien de causalité entre la catastrophe et le défaut de réaction de la majorité de la population contre certains actes du Gouvernement, le discours est de nature à insuffler superstition, intolérance et obscurantisme» et qu'«il finit ainsi par servir le prosélytisme et comporte dans son ensemble un ton offensif qui vise les "non-croyants", en même temps que le Gouvernement » ( $\$ 48)$.

Mais « si choquants et offensants qu'ils puissent être pour ceux qui ne partagent pas les croyances et opinions du public auquel ils sont adressés, les propos du requérant n'incitent pas à la violence et ne sont pas de nature à fomenter la haine contre les personnes qui ne sont pas membres de la communauté religieuse à laquelle appartient le requérant» et la sanction est jugée disproportionnée. En l'espèce, la désapprobation de la Cour à l'égard de ces assertions est palpable. Mais elle est tenue par l'application des critères très larges qu'elle établit pour la censure d'affirmation prenant place dans un débat d'idées ou politique. La conclusion eut sans doute été différente si la sanction infligée par les autorités turques avait été moins lourde.

Dans l'affaire Erbakan, la Cour juge que les propos de cet «homme politique notoire lors d'un rassemblement public révèlent [...] une vision de la société structurée exclusivement autour des valeurs religieuses et paraissent ainsi difficilement conciliables avec le pluralisme qui caractérise les sociétés actuelles où se confrontent les groupes les plus divers » et qu'en « usant d'une terminologie religieuse, l'orateur réduit notamment cette diversité, inhérente à toute société, en un simple clivage entre "croyants" et "non-croyants", et il lance un appel dans le but de former une ligne politique sur la base de l'appartenance religieuse ».

Mais elle conclut que «les poursuites pénales dirigées contre un homme politique quatre ans et cinq mois après la prétendue diffusion des propos incriminés ne représentaient pas un moyen raisonnablement proportionné aux buts légitimes visés, compte tenu de l'intérêt de la société démocratique à assurer et à maintenir le libre jeu du débat politique ${ }^{45}$.
Le résultat est identique dans l'affaire Varli, s'agissant de requérants ayant souhaité attirer l'attention sur le problème kurde et qui «s'exprimaient en leur qualité d'hommes de religion et membres d'un parti politique, dans le cadre de leur rôle d'acteurs de la vie sociale turque, n'incitant ni au recours à la violence ni à la résistance armée ni même au soulèvement, et qu'il ne s'agit pas d'un discours de haine, ce qui est aux yeux de la Cour l'élément essentiel à prendre en considération ${ }^{46}$.

La qualité d'hommes de religion ne semble pas déterminante dans cette affaire si ce n'est qu' elle explique la teneur du message (de paix) délivré sur le fondement de versets du Coran. En revanche, l'appel lors d'une conférence de presse donnée par un maire, personnage politique influent membre du Refah comme M. Erbakan, à «la désobéissance aux lois laïques de l'État turc» et " appelant les citoyens à préserver "[...] la hargne, la rancune, la haine qu'ils ont en eux" " peut être lourdement sanctionné sans violer l'article 10 de la Convention ${ }^{47}$.

En définitive, le bilan est assez équilibré et nulle supériorité de la protection d'une liberté sur l'autre ne se dessine nettement. L'arbitrage entre ces deux libertés éminentes reposant sur une grande marge d'appréciation des États n'est pas si défavorable à la liberté d'expression qu'on pourrait le penser à la lecture des quelques arrêts qui ont suscité de légitimes interrogations (Otto-Preminger, Wingrove). La surprotection de la Cour apparaît ainsi limitée aux critiques injurieuses reposant sur des mises en perspective obscènes de personnages clés d'une doctrine religieuse n'ayant pas (Dieu) ou plus (Jésus, Marie, sainte Thérèse, Mahomet) de corps physique. Rares sont ceux qui accèdent à ce statut de leur vivant. Tel n'est pas le cas de l'archevêque de l'affaire Klein. Il eut été intéressant de voir ce qu'aurait jugé la Cour si, dans l'affaire VBK, la plainte avait visé la représentation injurieuse de mère Teresa, décédée en 1997 et béatifiée en 2003, et non un membre d'un parti d'extrême droite. L'entrée de la religion dans l'arène politique ou médiatique ne lui confère pas non plus un statut particulier; le discours religieux y est protégé, malgré des apparences parfois trompeuses, comme n'importe quelle idée ou opinion. Entre ceux qui souhaiteraient manifestement que les convictions religieuses ne jouissent d'aucune protection et ceux qui aspirent à leur sanctuarisation au regard de toute critique ou moquerie, la Cour a, semble-t-il, trouvé un juste milieu qui auréole ses juges des vertus laïques que la Cour magnifie par ailleurs (neutralité, égalité, tolérance). 
